\title{
Técnicas de marketing en las cuentas oficiales de Twitter de los artistas flamencos
}

\author{
Marketin teknikak artista flamenkoek \\ Twitter-en dituzten kontuetan \\ Marketing techniques in the official \\ Twitter accounts of flamenco artists \\ Rafael Cano Tenorio* \\ Universidad de Cádiz
}

RESUMEN: El arte flamenco ha sido catalogado como patrimonio inmaterial de la humanidad por la UNESCO. En la presente investigación, se analizan las diferencias en la publicación de contenido en las cuentas oficiales de nueve artistas flamencos de gran reconocimiento en la red social Twitter. La metodología del estudio se ha basado el análisis de contenido, y se trata averiguar el grado de utilización de los contenidos de marketing según cada emisor analizado. Los resultados del estudio sirven para la mejora de la planificación estratégica en la comunicación digital de los artistas en el entorno digital.

PALABRAS CLAVE: Análisis de contenido, flamenco, Twitter, marketing, música.

ABSTRACT: The flamenco art has been catalogued as an Intangible Cultural Heritage of Humanity by UNESCO. This investigation analyses the differences in the publication of content in the Twitter's official accounts of nine recognized flamenco artists. The methodology of the case is based on the analysis content, and tries to find out the degree of use of the different categories of marketing contents according to each issuer analyzed. The results of the study attend for the strategic planning of the artists in the digital communication.

KEYWORDS: analysis of content, flamenco, Twitter, marketing, music.

* Correspondencia a / Corresponding author: Rafael Cano Tenorio. Universidad de Cádiz. Departamento de Marketing y Comunicación de la Universidad de Cádiz. Avda. de la Universidad, 4-11406 Jerez de la Frontera (Cádiz) - lete@jerez.es - http://orcid.org/0000-0003-3048-4141

Cómo citar / How to cite: Cano Tenorio, Rafael (2019). «Técnicas de marketing en las cuentas oficiales de Twitter de los artistas flamencos»; Zer, 24(46), 93-110. (https://doi.org/10.1387/zer.20319).

Recibido: 29 octubre, 2018; Aceptado: 27 abril, 2019.

ISSN 1137-1102 - eISSN 1989-631X / (c) 2019 UPV/EHU

(c) (i) Esta obra está bajo una licencia

Creative Commons Atribución 4.0 Internacional 


\section{Introducción}

Los orígenes del arte flamenco se remontan al siglo xix en la región de Andalucía, según Infante (1933), a partir del concepto de Folklore Andaluz, cuya fundación se debe a Antonio Machado en 1881'ㄹ, y el interés suscitado en los pensadores, escritores y musicólogos en torno al organismo estético, aunque casi exclusivamente en el aspecto de su expresión en torno a la poesía lírica. Según De la Plata (2005) es un arte exaltado por grandes artistas universales, como Federico García Lorca.

Esta disciplina musical ha sido reconocida por la UNESCO en el año 2010 como una expresión artística Patrimonio Cultural Inmaterial de la Humanidad. Según la prestigiosa organización, es un signo de identidad de numerosos grupos y comunidades, especialmente de la comunidad étnica gitana que ha desempeñado un papel esencial en la evolución de este arte universal. Dentro de los tipos de artistas flamencos se identifican varias disciplinas, cada una con sus características singulares. Según Infante (1933), con respecto al papel que realizan existen tres tipos: cante, baile, y toque. El Flamenco está en continua evolución, según el autor Steingress (2004). Para González (2015: 145), el arte Flamenco, como hecho cultural, ha estado expuesto al cambio, aunque la flamencología tradicionalista nos ha presentado este arte como un producto estático. Según Ríos (2011: 6), el Flamenco nunca fue más rico en tendencias que en la actualidad, en cualquiera de sus disciplinas artísticas, pues es un arte en constante evolución y mestizaje. Sin embargo, según Homann (2015: 39) no todo en el flamenco actual es creación.

La transmisión del flamenco se efectúa en el seno de dinastías de artistas, familias, peñas de flamenco y agrupaciones sociales, que desempeñan un papel fundamental y necesario en la preservación y difusión del mismo. González (2010) señala que «el flamenco constituye uno de los marcadores más evidentes de la identidad andaluza» (p. 145). Hay que tener en cuenta que un aspecto estrechamente ligado a la estructuración interna del campo flamenco, según Aix (2002: 120), es el territorio. La ciudad constituye el territorio clásico de la industria cultural y así ocurre con el flamenco en Andalucía, donde ciudades como Sevilla y Jerez, según Cerezo (2015: 70) concentran gran parte del tejido industrial relacionado con este arte. Aunque según el autor Steingress (2002: 49) el flamenco actualmente no es sólo una manifestación popular de la cultura de la región de Andalucía únicamente, sino que va mucho más allá de sus fronteras. Una de las grandes particularidades en los públicos del flamenco, según expone Giguère (2005), es la convivencia en plena armonía entre personas de raza gitana y "gachos» (personas que no son de raza gitana). La conexión entre gitanos y el complejo de arte

\footnotetext{
1 Antonio Machado Álvarez fue miembro fundador y secretario de la sociedad Folk-lore andaluz, que tenía por objeto la conservación y publicación de las tradiciones populares andaluzas.
} 
flamenco según Aoyama (2007: 106) se ha acentuado con la profesionalización del arte flamenco. Hoy en día la participación de artistas de las tres disciplinas flamencas (bailarines, cantantes y guitarrista) que no son gitanos es bastante significativa, y la tendencia es que cada vez sea mayor.

En los últimos años, socialmente los artistas flamencos han cobrado enorme influencia, debido en parte a la repercusión mediática de todas las actividades en las que participan, más allá de las que guardan relación con el desarrollo de las su profesión. La comunicación, en este sentido, tiene un rol de gran importancia en la relación de los artistas flamencos con el entorno que les rodea.

Con respecto a las referencias bibliográficas del arte Flamenco, según Aix (2002: 110), es importante señalar que cuenta, como campo artístico formado, con una literatura bastante autorreferencial y en su mayoría ensimismada y desarrollada por autores más próximos al encomio que a la autocrítica.

Según De la Plata (2005: 8), en el final del Siglo XviII y durante el Siglo XIX aparecieron los primeros investigadores del cante flamenco, destacando Antonio Machado y Álvarez, «Demófilo», considerado como el «el primer flamencólogo español».

En los últimos tiempos en el ámbito académico se ha avanzado mucho en el estudio del flamenco, llegando incluso a implantarse en al año 2018 un título oficial con un programa de estudios sobre el Flamenco en la Universidad de Cádiz, con el Máster Interuniversitario en Investigación y Análisis del Flamenco. También hay que destacar, según López (2010), la puesta en funcionamiento del Doctorado de Flamenco en la Universidad de Sevilla.

\subsection{Flamenco, contenidos y estrategias de marketing en el entorno DIGITAL}

A diferencia de otros períodos históricos anteriores, el autor Steingress (2008: 255) señala que la música se ha convertido a principios del siglo XXI en un bien cultural que se difunde y proyecta en todos los sectores sociales, aunque su diversificación obedece a determinados factores sociales y culturales, sobre todo la edad y el nivel de estudios.

Dentro del ámbito cultural, según Cano y Rostoll (2018: 42), las nuevas tecnologías han avanzado mucho en los últimos tiempos. La difusión del arte flamenco, según Aoyama (2007: 104), se ha visto reforzada gracias a las nuevas tecnologías, al igual que sucede en otros entornos artísticos. 
El flamenco, desde la visión de Parra (2012: 53), es ante todo comunicación porque transmite sentimiento y emociones. Teniendo en cuenta lo anterior, y en consonancia con el desarrollo de las estrategias de comunicación en el entorno del arte Flamenco, Aix (2002) subraya que aumentan los profesionales flamencos especializados: artistas, managers, productores, gestores, periodistas, escritores, académicos, entre otros.

Internet puede considerarse como medio fundamental para el cambio y desarrollo de los profesionales flamencos. Según Steingress(2004), el efecto más llamativo es que el flamenco ha dejado de ser una manifestación solo de lo local, para llegar a ser un objeto mercantil con etiqueta de música étnica con proyección internacional. En los últimos años Internet ha sufrido una revolución en todos los sentidos. El medio Internet influye decisivamente, y según Perujo (2015), Internet «ha funcionado como motor y como espejo dentro un proceso de internacionalización ya lanzado, que ahora ha encontrado su propio sitio en el universo online» (p. 75). Según Moreno (2015), un factor de creatividad fundamental en los actores culturales en el entorno digital es la redefinición. Según Patricio y Gallo (2015: 113), la mejor forma de llegar a los públicos es la integración de las acciones de comunicación.

El flamenco tiene audiencias no sólo a nivel nacional en su país de origen, que es España. Aoyama (2007: 111) señala que existen públicos en muchos rincones del mundo, destacando países como Estados Unidos o Japón, entre otros.

La elaboración de estrategias relacionadas con la comunicación y el marketing en la trayectoria de los artistas conlleva la construcción de una personalidad artística que se apropia, según Aix (2002: 122), en relación a sus posibilidades y en orden a las reglas del campo, de atributos de las diferentes tradiciones, lo que se llama patrimonialización y usos del patrimonio. Las estrategias individuales, según Kyle (2017: 17) pueden incluir salir de un endogrupo para unirse a otro grupo que produce una identidad social más positiva. El flamenco está en constante evolución, y se relaciona con otras disciplinas musicales mezclándose.

En relación al flamenco como objeto de mercado, es un arte que genera, según Lasso de la Vega (2005), mayor atractivo comercial, y contribuye también a engrosar el universo simbólico de lo andaluz como el espacio de la magia, el duende o el misterio, claras expresiones según la autora, de la espontaneidad y la irracionalidad.

\subsection{TWITTER COMO HERRAMIENTA DE COMUNICACIÓN DIGITAL}

El desarrollo de estrategias de comunicación y marketing online ha obligado a los artistas de todas las disciplinas flamencas a poner en marcha acciones para una mejor gestión de su imagen, de cara a sus diferentes audiencias. Los estudios que re- 
lacionan la comunicación y la música, y más concretamente el flamenco, han proliferado en los últimos años de manera significativa. Hambrick (2012) afirma que existen usuarios influyentes que difunden información a través de la red social Twitter, herramienta de comunicación digital fundamental para la difusión de contenidos, que pueden guardar relación con el marketing. Los artistas flamencos pueden aprovecharse de la gestión de estas herramientas para explotar todas las posibilidades.

Como indican Colom, Solá y Micó (2018: 182), las herramientas del marketing han podido contribuir a la proyección y cristalización de una determinada escena musical. Según estos autores, más allá de la relación de los artistas con la industria, tienen como finalidad compartir una obra artística con sus seguidores. Al mismo tiempo, están comprobando la eficacia de las herramientas que ofrecen las nuevas tecnologías de la comunicación.

En todo caso los responsables en gestionar las cuentas oficiales de los artistas deben tener planes de acción detallados. Salazar y Prieto (2015: 21) señalan que «toda institución que quiera lograr una comunicación efectiva en Twitter debe elaborar un plan de comunicación en el que se definan los objetivos y las acciones de comunicación que se llevarán a cabo».

La buena explotación de la comunicación y el marketing digital permite a los artistas del mundo flamenco diferenciarse del resto de sus competidores, ya sean del ámbito del arte flamenco o no. Los contenidos flamencos, según Arjona (2015: 323), encuentran igual amoldamiento en los medios impresos que en los medios virtuales que se alojan en el medio Internet. Perujo (2015: 76) señala que se cuentan por miles los sitios web del flamenco en Internet, pues las páginas webs, los blogs y la presencia en redes sociales de instituciones, empresas, compañías, peñas, artistas, investigadores, aficionados y demás integrantes de la amplia sociología flamenca, y entre todos han acrecentado la internacionalización del arte. Según García y López (2016: 130), en el contexto de la sociedad actual las redes sociales se destapan como un instrumento válido, en tanto en cuanto tiene un gran potencial de conexión con el público por un uso cada vez más importante de una capa amplia de población. El autor Steingress (2008: 249) recalca que pese a que las nuevas tecnologías de la comunicación pueden cambiar nuestras formas de vida cotidiana, el actual cambio de hábitos culturales surge de un complejo entramado de muy diferentes factores que influyen a nivel global y en función de las nuevas necesidades y estilos de vida, cambiando incluso las mentalidades.

«En los últimos años el flamenco, empujado por las nuevas tecnologías, ha ido modificando la forma en la que se presenta al público; además de una manifestación cultural de ámbito universal, es una oportunidad de negocio de una magnitud inimaginable, no sólo a escala nacional, sino también internacional.» (Hurtado y Gordillo, p. 39) 
El mundo del arte flamenco cuenta con un conjunto de relaciones entre diferentes públicos y audiencias con muchas singularidades propias, y que a diferencia de otros campos, son artísticos o de otro ámbito.

El concepto de público, según Capriotti (2009), es uno de los ejes fundamentales en el ámbito del marketing, poniéndose de manifiesto por parte de investigadores y profesionales como un concepto de vital importancia para las organizaciones.

Según Ríos (2011), el público en el arte flamenco crece cada vez más, es heterogéneo y le gusta lo tradicional y lo experimental.

Micaletto y Cano (2018) señalan que en el entorno digital las acciones de relaciones públicas son empleadas por los emisores de contenidos digitales para difundir ante sus públicos una imagen adecuada a sus fines y actividades. Y en este caso el arte Flamenco no se queda atrás. Thelwall y Cugelman (2017) afirman que los nuevos medios, como por ejemplo la red social Twitter, pueden distribuir mensajes a una gran audiencia a la que sería difícil llegar por otros medios.

\section{Metodología de la investigación}

La técnica metodológica principal y fundamental que se ha utilizado en este estudio ha sido el análisis de contenido. Con respecto a la misma, hay que recalcar que «supone aplicar sistemáticamente unas reglas fijadas previamente que sirvan para medir la frecuencia con que aparecen unos elementos para estudiar algunos de los aspectos que nos parecen útiles conforme a los propósitos de nuestra investigación» (Berganza y Ruiz, 2005: 213).

El análisis de contenido es un conjunto de procedimientos interpretativos, y según el autor Piñuel (2002), de productos comunicativos que proceden de procesos singulares de comunicación previamente registrados, y basados en técnicas de medida, a veces cuantitativas, y a veces cualitativas. Según el mismo autor no existe un modelo único de realización del mismo. La finalidad que se persigue con esta técnica metodológica, según Berganza y Ruiz (2005), es la aproximación al objeto de estudio.

\subsection{DesCripCión DEL PROCESO DE INVESTIGACióN}

La metodología de investigación del presente estudio ha seguido la siguiente plantilla de análisis: 
TABLA 1

Plantilla de análisis de la investigación

\begin{tabular}{|c|l|}
\hline Etapa I & $\begin{array}{l}\text { Localización de las direcciones oficiales de las cuentas oficiales de los artistas } \\
\text { flamencos en la red social Twitter }\end{array}$ \\
\hline Etapa II & Acceso a la cuenta oficial en Twitter de cada artista flamenco \\
\hline Etapa III & Selección de los artistas flamencos que conforman la muestra de la investigación \\
\hline Etapa IV & $\begin{array}{l}\text { Registro de emisión de contenido: anotación de dirección URL, título, fecha y } \\
\text { hora de acceso }\end{array}$ \\
\hline Etapa V & Introducción de los contenidos emitidos en fichas de análisis \\
\hline Etapa VI & Clasificación y categorización de los contenidos emitidos \\
\hline Etapa VII & Introducción de los contenidos emitidos en hojas de cálculo \\
\hline Etapa VIII & Análisis de los datos del estudio \\
\hline Etapa IX & Redacción de los resultados del estudio \\
\hline
\end{tabular}

Fuente: elaboración propia.

\subsection{SelecCiÓN DE LA MUESTRA EN LA INVESTIGACiÓN}

Como demuestra la evolución histórica del arte flamenco desde sus orígenes a partir de mediados del siglo XIX, según señala Steingress (2004), las tendencias de transgresión estética y de fusión con otros tipos de músicas tradicionales y populares no son precisamente sólo una característica del flamenco contemporáneo. El flamenco apareció ya como un producto más de una serie de fusiones en la música romántica.

La importancia de la selección del tamaño de la muestra de la investigación, según Krippendorff (1990), es una cuestión relacionada con la dificultad-beneficio, y depende en gran medida de la forma en que se distribuye en la muestra el atributo que se desea generalizar.

La elección de los artistas flamencos, en este estudio, se basa principalmente en su alcance mediático en la red social que se analiza, y la pertenencia de los artistas a alguna de las disciplinas flamencas propuestas en el estudio, según la clasificación de Infante (1933): cante, baile y toque. Aix (2002: 114) señala: «El análisis de un campo cultural como el flamenco pasa por reconocer su origen y grado de autonomía, es decir, la independencia con respecto a otros campos con los que está relacionado». Para identificar a los artistas flamencos hay que tener en cuenta al autor de la Cátedra de Flamencología Ríos (2011: 7), que señala que en el siglo xxi, y pese a su mezcla con otras músicas, siempre sobresale lo flamenco en la fusión que se produce. 
El período de investigación comprende desde el 1 de junio de 2017 al 31 de diciembre de 2017, y se ha registrado la emisión de contenido que realiza cada artista flamenco en la red social Twitter, realizado en enero de 2017.

Teniendo en cuenta los criterios para identificar qué artistas pueden ser considerados flamencos, y con respecto al análisis de los contenidos emitidos en la red social analizada, se han seleccionado un total de 9 artistas flamencos, con mayor o menor nivel de mestizaje con otras disciplinas artísticas musicales, pero entendiendo, ante todo, que todos son principalmente artistas flamencos.

En este sentido, el investigador en Flamencología Ríos (2011) afirma:

«en las fusiones del flamenco con el jazz, el pop, o el rock y rap. El Flamenco siempre prevalece, como antaño prevaleció al fusionarse con la farruca, el garrotín, el punto cubano, la vidalita, la milonga, la ranchera o la guajira» (p. 11).

Según García Plata (1995), el flamenco es una expresión musical que nació de diversos orígenes, cuya fuerza es precisamente esa capacidad de asimilar otros géneros musicales (p. 30)

Teniendo en cuenta lo expuesto anteriormente, la selección definitiva para la investigación de artistas del arte flamenco en la disciplina de Cante ha sido:

—Diego Ramón Jiménez Salazar, «El Cigala»

- Jennifer Jessica Martínez Fernández, «India Martínez».

- María Rosa García García, «Niña Pastori».

En el caso de los artistas flamencos de la disciplina de Baile, la selección ha sido:

—Eva María Garrido, conocida artísticamente como «Eva Yerbabuena».

— Antonio Fernández Montoya, conocido como «Farruco» o «El Farru».

- Sara Pereyra Baras, conocida en ámbitos artísticos como «Sara Baras».

En tercer lugar, se presenta la selección de los artistas flamencos de la disciplina de Toque:

—Diego Moreno Jiménez, «Diego del Morao».

- Francisco López Cepero García, «Paco Cepero».

- Vicente Amigo Girol. 


\subsection{Categorización de los datos obtenidos EN El PROCESO DE INVESTIGACIÓN DE CONTENIDOS}

El contenido emitido en la red social Twitter desde las cuentas oficiales de los artistas, se ha clasificado en fichas de análisis, en las siguientes clases y categorías de contenido:

TABLA 2

Categorización de los datos obtenidos

\begin{tabular}{|c|l|}
\hline $\begin{array}{c}\text { Categoría de } \\
\text { contenido }\end{array}$ & \multicolumn{1}{|c|}{ Descripción } \\
\hline $\begin{array}{c}\text { Contenidos de } \\
\text { la carrera artística }\end{array}$ & $\begin{array}{l}\text { Contenido publicado con relación a la carrera del artista flamenco. Se incluyen } \\
\text { las nominaciones a premios, lanzamientos y publicaciones, relación con } \\
\text { productoras y discográficas, y actuaciones en conciertos y festivales. }\end{array}$ \\
\hline $\begin{array}{c}\text { Contenidos } \\
\text { de marketing }\end{array}$ & $\begin{array}{l}\text { Publicaciones que intentan persuadir a los seguidores para que compren } \\
\text { productos de las marcas que patrocinan a los artistas musicales. Se incluyen } \\
\text { compromisos comerciales y promociones relacionadas con sorteos para la } \\
\text { promoción de la cuenta oficial en Twitter de los artistas. También se incluyen } \\
\text { en estas categorías anuncios sobre lanzamientos y publicaciones de otros artistas. }\end{array}$ \\
\hline $\begin{array}{c}\text { Contenidos de vida } \\
\text { personal }\end{array}$ & $\begin{array}{l}\text { Publicaciones referidas a información sobre la vida privada de los artistas } \\
\text { flamencos: amigos, familia, compañeros de trabajo, ocio, pensamientos, tiempo } \\
\text { libre, etc. }\end{array}$ \\
\hline Contenidos sociales & $\begin{array}{l}\text { Contenido referido a la relación de los artistas flamencos con sus seguidores. Se } \\
\text { incluyen contenidos de participación en campañas sociales o institucionales de } \\
\text { los artistas flamencos. }\end{array}$ \\
\hline
\end{tabular}

Fuente: elaboración propia.

\section{Utilización de técnicas de márketing en las cuentas oficiales en Twitter de los artistas flamencos}

Una vez monitorizados todos los datos, se comprueba que se han analizado en la investigación un total 1.527 publicaciones, emitidas en la red social Twitter, de las que 44 fueron contenidos de marketing. El resto de publicaciones se desglosan de la siguiente manera: 1.131 publicaciones de contenidos de la carrera artística, $185 \mathrm{pu}-$ blicaciones de contenidos sociales, y 167 publicaciones de contenidos sobre la vida personal de los artistas flamencos. 


\subsection{UTILIZACIÓN DE TÉCNICAS DE MARKETING EN LAS CUENTAS OFICIALES EN TwitTer De los artistas FlamenCOS DE LA DisCiPLina DE Baile Flamenco}

Los artistas de esta disciplina emitieron un total de 497 publicaciones, de las que 22 eran contenidos de marketing. El resto de publicaciones se desglosan de la siguiente manera: 355 publicaciones sobre contenidos de la carrera artística de los bailaores, 60 de la vida personal, y 60 de contenidos sociales.

\section{TABLA 3}

Emisión de contenidos en las cuentas oficiales de los artistas flamencos de la disciplina de Baile

\begin{tabular}{|c|c|c|c|c|}
\hline Artista Flamenco & $\begin{array}{c}\text { Contenidos de la } \\
\text { Carrera Artística }\end{array}$ & $\begin{array}{c}\text { Contenidos de } \\
\text { Marketing }\end{array}$ & $\begin{array}{c}\text { Contenidos de } \\
\text { Vida Personal }\end{array}$ & $\begin{array}{c}\text { Contenidos } \\
\text { Sociales }\end{array}$ \\
\hline Eva Yerbabuena & 92 & 1 & 20 & 11 \\
\hline Farruco & 96 & 1 & 8 & 4 \\
\hline Sara Baras & 167 & 20 & 32 & 45 \\
\hline
\end{tabular}

Fuente: elaboración propia.

Eva Yerbabuena emitió un total 124 publicaciones, de las cuales una era de contenido de marketing. El resto de publicaciones emitidas por la artista nacida en Fráncfort fueron 92 publicaciones de la carrera artística, 20 de la vida personal, y 11 de contenidos sociales.

El bailaor flamenco Farruco emitió un total de 109 publicaciones en el período de análisis, de las cuales solo una fue de contenido sobre marketing. El resto de publicaciones fueron 96 de la carrera artística, 8 de contenidos de la vida personal, y 4 de contenidos sociales en los que se implica el artista.

La artista isleña Sara Baras publicó un total de 264 publicaciones, de las cuales 20 fueron de contenido de marketing. El resto de publicaciones de la artista se desglosan de la siguiente manera: 167 publicaciones de la carrera artística de la bailaora, 32 de la vida personal, y 45 de contenidos sociales. 


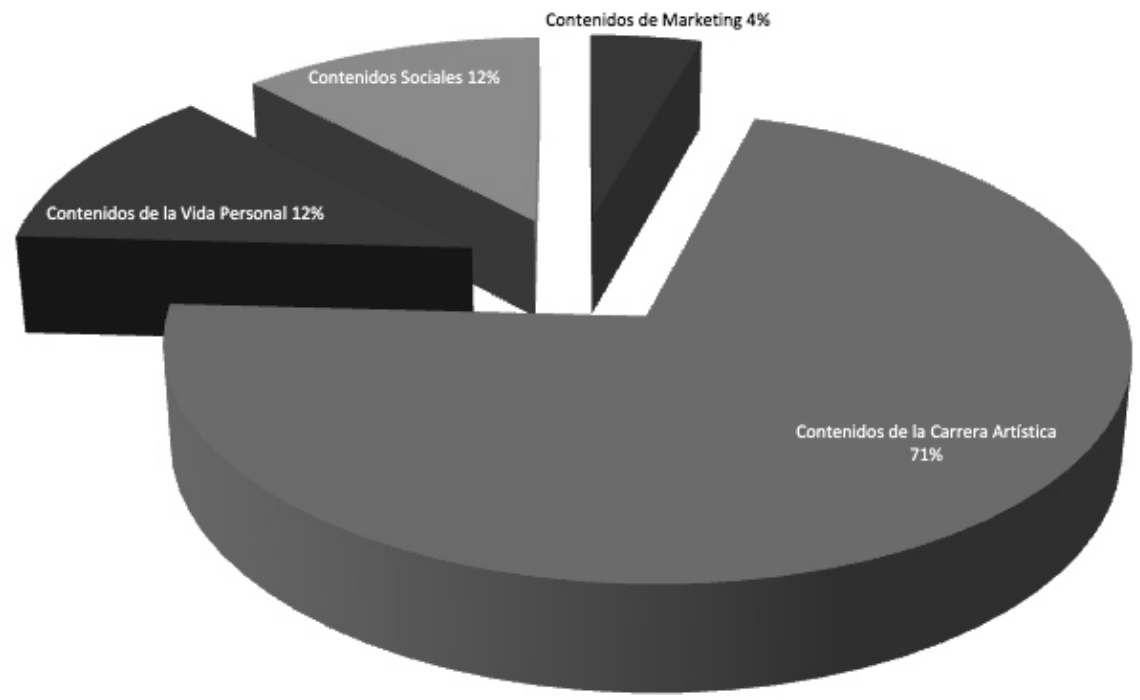

Figura 1

Emisión de contenidos en las cuentas oficiales de los artistas flamencos de la disciplina de Baile

Fuente: elaboración propia.

\subsection{UTILIZACión DE tÉCNiCAS DE MARKETING eN LAS CUENTAS OfiCIALES en Twitter de los artistas flamencos de la disciplina de Cante FLAMENCO}

Los artistas de la disciplina de Cante Flamenco emitieron un total de 832 publicaciones, de las que 22 eran contenidos de marketing. El resto de publicaciones se desglosan de la siguiente manera: 609 publicaciones sobre contenidos de la carrera artística de los bailaores, 87 de la vida personal, y 114 de contenidos sociales.

\section{TABLA 4}

Emisión de contenidos en las cuentas oficiales de los artistas flamencos de la disciplina de Cante

\begin{tabular}{|c|c|c|c|c|}
\hline Artista Flamenco & $\begin{array}{c}\text { Contenidos de la } \\
\text { Carrera Artística }\end{array}$ & $\begin{array}{c}\text { Contenidos de } \\
\text { Marketing }\end{array}$ & $\begin{array}{c}\text { Contenidos de } \\
\text { Vida Personal }\end{array}$ & $\begin{array}{c}\text { Contenidos } \\
\text { Sociales }\end{array}$ \\
\hline Diego «El Cigala» & 171 & 2 & 2 & 9 \\
\hline India Martínez & 376 & 18 & 32 & 100 \\
\hline Niña Pastori & 62 & 2 & 3 \\
\hline
\end{tabular}

Fuente: elaboración propia. 
Diego «El Cigala» publicó un total de 184 publicaciones en la red social Twitter en el período de análisis, de las que 2 fueron contenidos de marketing. El resto de publicaciones del artista se dividieron de la siguiente manera: 171 publicaciones de la carrera artística, 2 de la vida personal, y 9 de contenidos sociales.

India Martínez emitió un total de 576 publicaciones, de las que 18 publicaciones fueron de contenidos que utilizan técnicas de marketing. El resto de publicaciones de la artista nacida en Córdoba se distribuyeron de la siguiente manera: 376 publicaciones sobre la carrera artística, 82 de la vida personal, y 100 de contenidos sociales.

La isleña Niña Pastori emitió un total de 72 publicaciones, de las que 2 fueron de contenidos que utilizan técnicas de marketing. El resto de publicaciones se distribuyeron de manera que 62 fueron de la carrera artística, 2 de la vida personal de la artista, y 5 de contenidos sociales.

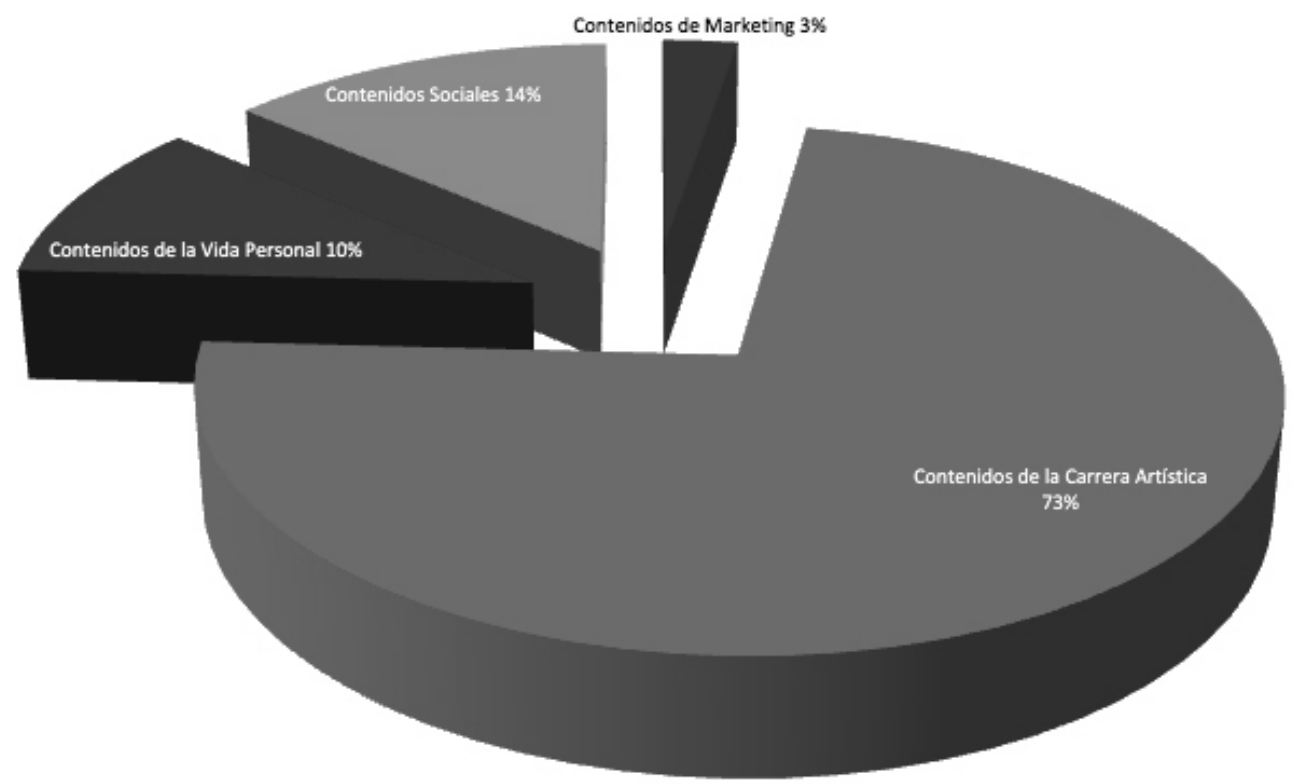

Figura 2

Emisión de contenidos en las cuentas oficiales de los artistas flamencos de la disciplina de Cante

Fuente: elaboración propia. 


\subsection{UTILIZACIÓN DE TÉCNICAS DE MARKETING EN LAS CUENTAS OFICIALES en TwitTer de los artistas flamencos de la disciplina de Toque FlamenCO}

Los artistas de esta disciplina emitieron un total de 198 publicaciones, de las que ninguna era de marketing. El resto de publicaciones se desglosan de la siguiente manera: 167 publicaciones sobre contenidos de la carrera artística de los bailaores, 20 de la vida personal, y 11 de contenidos sociales.

TABLA 5

Emisión de contenidos en las cuentas oficiales de los artistas flamencos de la disciplina de Toque

\begin{tabular}{|c|c|c|c|c|}
\hline Artista Flamenco & $\begin{array}{c}\text { Contenidos de la } \\
\text { Carrera Artística }\end{array}$ & $\begin{array}{c}\text { Contenidos de } \\
\text { Marketing }\end{array}$ & $\begin{array}{c}\text { Contenidos de } \\
\text { Vida Personal }\end{array}$ & $\begin{array}{c}\text { Contenidos } \\
\text { Sociales }\end{array}$ \\
\hline Diego del Morao & 26 & 0 & 6 & 5 \\
\hline Paco Cepero & 102 & 0 & 14 & 5 \\
\hline Vicente Amigo & 39 & 0 & 0 & 1 \\
\hline
\end{tabular}

Fuente: elaboración propia.

Diego del Morao publicó un total de 37 contenidos en el período de análisis, de los cuáles ninguno utilizó técnica de marketing. El resto de contenidos del artista jerezano trataba sobre su carrera artística en un total de 26 publicaciones, 20 sobre su vida personal, y 11 sobre contenidos sociales.

El otro artista de la disciplina de Toque Flamenco jerezano analizado en el estudio es Paco Cepero, que emitió un total de 121 publicaciones en el período en el que se realizó el estudio, y de los cuales ninguna utilizó técnicas de marketing. El resto de publicaciones del artista se distribuyeron de la siguiente manera: 102 publicaciones sobre la carrera artística, 14 de la vida personal, 5 de contenidos sociales.

Vicente Amigo emitió un total de 40 publicaciones en la red social Twitter en el período de análisis, de las cuales ninguna utilizó técnicas de marketing. El resto de publicaciones del artista nacido en Guadalcanal se distribuyeron de la siguiente manera: 167 de la carrera artística, 32 de la vida personal, y 11 de contenidos sociales. 


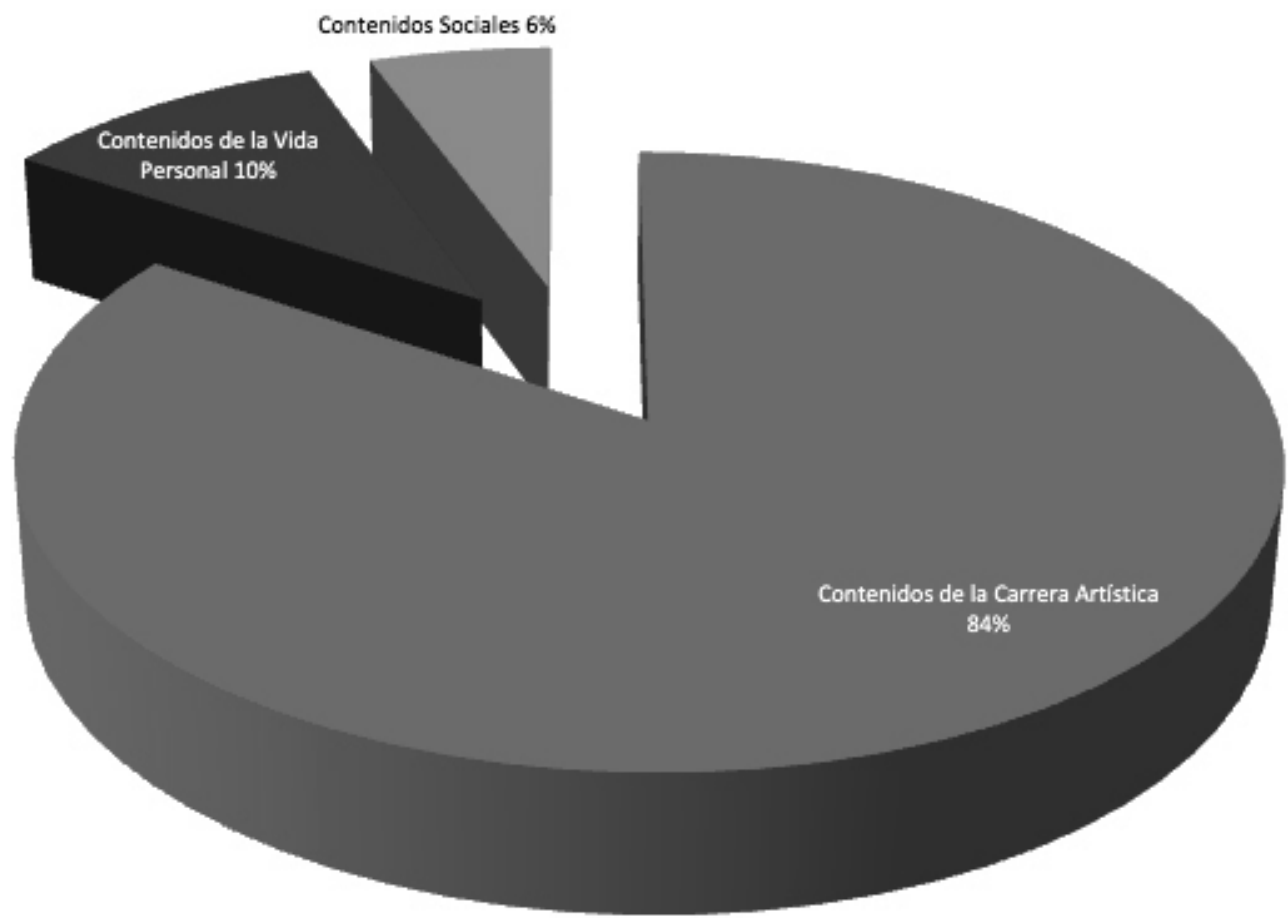

FIGURA 3

Emisión de contenidos en las cuentas oficiales de los artistas flamencos de la disciplina de Toque

Fuente: elaboración propia.

\section{Conclusiones}

Twitter es una importante herramienta de marketing, pudiendo ser explotada por artistas de toda índole, incluidos los artistas flamencos. De todas las publicaciones monitorizadas, solo un 2,88\% fueron de contenidos de marketing.

Se observa diferente comportamiento en los artistas de cada disciplina flamenca (Baile, Cante y Toque), pues emiten de diferente cantidad de contenido en la red social Twitter. Los artistas pertenecientes a la disciplina de Cante Flamenco han emitido un total de 832 publicaciones, mientras que los de Baile Flamenco 497. Muy atrás en cuanto a número de publicaciones quedan los artistas de la disciplina de Toque.

Los artistas flamencos que más contenido emiten en total son de género femenino, la artista cordobesa India Martínez, con el 37,72\% de las publicaciones 
totales monitorizadas, y la bailaora isleña Sara Baras con el 17,28\% de las publicaciones totales monitorizadas en el estudio. Hay que reseñar, por el contrario, que los artistas flamencos analizados con menos contenido emiten son hombres de la disciplina de Toque Flamenco, el jerezano Diego del Morao con el 2,42\% del total de las publicaciones monitorizadas, y Vicente Amigo con el 2,61\% de las publicaciones totales.

En el estudio se constata un gran desarrollo de este medio de comunicación en el mundo del arte flamenco en los últimos tiempos, pero también que aún queda mucho trabajo por hacer, ya sea por falta de planificación estratégica en marketing y comunicación por parte de los artistas al emitir contenido, o por falta de profesionalización de las personas a las que se encomienda estas tareas, a la hora de publicar contenido en Twitter.

En cuanto al porcentaje de emisión de contenidos de marketing sobre el total, destacan los artistas de la disciplina de Baile Flamenco, pues el 4,42\% de publicaciones que emiten lo hacen utilizando técnicas de marketing en sus contenidos. En este sentido, los artistas de la disciplina de Cante Flamenco emiten el 2,64\% de su contenido utilizando técnicas de marketing, mientras que los artistas de Toque Flamenco no utilizan contenidos de marketing.

Los artistas flamencos con mayor porcentaje de emisión de contenidos de marketing han sido Sara Baras con el 7,57\% de contenidos de marketing sobre su total de publicaciones, y India Martínez, con el 3,12\% de contenidos sobre su total de publicaciones.

Se ha observado en el estudio que los contenidos de marketing son poco utilizados y explotados por los artistas flamencos, y que se podrían realizar más acciones basadas en técnicas de marketing para mejorar los datos. Los artistas basan sus publicaciones en contenidos relaciones con su carrera artística, superando en todas las disciplinas más del 70\% sobre el total de las publicaciones que los artistas realizan.

Esta investigación puede servir para el desarrollo de futuras líneas de investigación en las que se puede trabajar, y que tengan relación con la comunicación, el marketing y el arte flamenco, y en las que se podrían abrir nuevos estudios de interés para la comunidad científica en relación al análisis de las audiencias del mundo del arte flamenco, o atendiendo a los efectos del mensaje sobre los receptores, según la clasificación de los autores Berganza y Ruiz (2005). 


\section{Bibliografía}

Aix Gracia, F. (2002). El arte flamenco como campo de producción cultural. Aproximación a sus aspectos sociales. Anduli, Revista Andaluza de Ciencias Sociales (1), pp. 109-125.

Aoyama, Y. (2007). The role of consumption and globalization in a cultural industry: The case of Flamenco. Geoforum, 38 (1) pp. 103-113.

Arjona Pabón, M. (2015). La crítica flamenca en los medios de comunicación escritos. El caso de la XIV y XV Bienal de Flamenco de Sevilla. Tesis doctoral. Universidad de Sevilla, España.

Berganza Conde, M. y Ruíz San Román, J. (2005). Investigar en Comunicación. Madrid: McGraw-Hill.

Cano Tenorio, R. y Rostoll Ariza, J. (2018). Uso de las redes sociales en museos internacionales. Revista de la Asociación Española de Investigación en Comunicación, 5(9), pp. 41-49.

Capriotti, P. (2009). Branding corporativo. Santiago de Chile: Libros de la Empresa.

Cerezo Gallego, A. (2015). La industria cultural del flamenco: aspectos económicos y fiscales. Tesis doctoral. Universidad de Málaga, España.

Colom Valls, I.; Solà Saña, S. y Micó Sanz, J. L. (2018). Estrategias y herramientas de marketing y comunicación y su eficacia en la proyección del pop y el rock en lengua catalana. adComunica. Revista Científica de Estrategias, Tendencias e Innovación en Comunicación, 16, pp. 179-199.

De la Plata, J. (2005). El Flamenco, Patrimonio intangible de la cultura andaluza. Revista de flamencología (21), pp. 5-12.

Patricio De Melo Cardos, A.T. y Gallo, P. (2014). La comunicación integrada y la narrativa transmedia. Revista de la Asociación Española de Investigación de la Comunicación, 1(1), pp. 112-116.

García Orosa, B. y López García, X. (2016). Las redes sociales como herramienta de distribución on line de la oferta informativa en los medios de España y Portugal. Zer, Revista de Estudios en Comunicación, 21(40), pp. 125-139.

García Plata, M. (1995). El Flamenco moderno, Camarón de la Isla: ¿Un cantaor revolucionario?. Revista de flamencología (2), pp. 29-44.

Giguère, H. (2005). El flamenco como patrimonio inmaterial de la humanidad, un estudio del fenómeno en Jerez de la Frontera. Revista Internacional Música Oral del Sur, 6, pp. 311-319.

González Martín, J. (2016). Basilio Martín Patino, Desde lo más hondo: una reflexión sobre tecnología y flamenco. Revista de Investigación sobre Flamenco (13), pp. 125-150.

Hambrick, M. (2012). Six Degrees of Information: Using Social Network Analysis to Explore the Spread of Information within Sport Social Networks. International Journal of Sport Communication, 5(1), pp. 16-34.

Homann, F. (2015). Una copla flamenca en el Siglo xxi: ¿Todavía tradicional y popular?. Revista de Investigación sobre Flamenco (12), pp. 35-74. 
Hurtado Guapo, M.A. y Gordillo Tapia, L.V. (2014). Análisis del color en los websites de productos sobre flamenco. Revista de Investigación Flamenco Telethusa 7((8), pp. 38-43.

Junta de Andalucía (2010). Reproducción facsímil de la edición de la Junta de Andalucía de 1980 de «Orígenes de lo flamenco y secreto del Cante jondo, 1929-1933». Sevilla: Consejería de Cultura de la Junta de Andalucía. < https://www.juntadeandalucia.es/ cultura/flamenco/content/or\%C3\%ADgenes-de-lo-flamenco-y-secreto-del-Cantejondo-de-blas-infante> (20-02-2018).

Kyle Chicotsky, B. (2017). The brand persona: operationalizing a synthesis of brand equity and social capital. Tesis doctoral. The University of Alabama, Estados Unidos.

Krippendorf, K. (1990). Metodología del análisis de contenido. Teoría y práctica. Barcelona: Paidós.

Lasso de la Vega González, M. (2005). El quejío andaluz. La conversión del objeto artístico en bien de consumo. I Jornadas de Flamenco y Comunicación, Universidad de Sevilla.

Lavanguardia.com (2018). El Flamenco tendrá un título oficial en la Universidad de Cádiz. <https://www.lavanguardia.com/local/sevilla/20180704/45657327708/el-flamencotendra-en-la-universidad-de-cadiz-un-titulo-oficial.html > (03-10-2018)

López Castro, M. (2010). La didáctica del Flamenco: Una aproximación a su historia y algunas propuestas de trabajo. Revista de Investigación sobre Flamenco (3), pp. 1-27.

Micaletto Belda, J. y Cano Tenorio, R. (2018). Relaciones públicas y Juego de Tronos: la imagen del Sevilla Fútbol Club, S.A.D. tras la visita de los actores al Sánchez Pizjuán. Zer, Revista de Estudios en Comunicación, 23(44), pp. 31-47.

Moreno Sánchez, I. (2015). Interactividad, interacción y accesibilidad en el museo transmedia. Zer, Revista de Estudios en Comunicación, 20(38), pp. 87-107.

Parra Pujante, A. (2012). El Flamenco a través de las teorías de la comunicación. Revista de Investigación sobre Flamenco (7), pp. 45-54.

Perujo Serrano, F. (2015). Flamenco y Marca España. En Cenizo Jiménez, José y Gallardo Saborido, Emilio (Ed.), Presumes que eres la ciencia (estudios sobre el flamenco) (pp. 46-79). Sevilla, España: Libros con Duende.

Piñuel Raigada, J. (2002). Epistemología, metodología y técnicas del análisis de contenido. Estudios de sociolingüística, 3 (1), pp. 1-42.

Ríos Ruiz, M. (2011). El flamenco ante el S.xxi: tradición y experimentalismo. Revista de flamencología (28), pp. 5-13.

Ruiz Olabuenaga, J. (2012). Teoría y práctica de la investigación cualitativa. Bilbao: Publicaciones de la Universidad de Deusto.

Salazar Puerta, S. y Prieto Dávila, P. (2015). Gestión y administración de la comunicación institucional en Twitter. Anuario Electrónico de Estudios en Comunicación Social «Disertaciones», 8, (1), pp. 11-26.

Sociedad Folk-lore andaluz (1882). El Folk-lore andaluz. Sevilla, España: Francisco Álvarez y C. ${ }^{a}$ Editores.

Steingress, G. (2002). El flamenco como patrimonio cultural o una construcción artificial más de la identidad andaluza. Anduli. Revista Andaluza de Ciencias Sociales, 1, pp. 43-64. 
Steingress, G. (2004). La hibridación transcultural como clave de la formación del Nuevo Flamenco (aspectos histórico-sociológicos, analíticos y comparativos). Trans. Revista Transcultural de Música, 8, pp. 1-33.

Steingress, G. (2008). La música en el marco del análisis de la cultura contemporánea: un replanteamiento teórico y metodológico. Política y Sociedad, 45(1), pp. 237-260.

Thelwall, M. y Cugelman, B. (2017). Monitoring Twitter Strategies to discover resonating topics: the case of the UNDP. El profesional de la información, 26 (4), pp. 649-661.

UNESCO. El flamenco. <https://ich.unesco.org/es/RL/el-flamenco-00363> (22-022018). 